\title{
Histological and immunohistochemical study of the effect of omega-3 fatty acids on ifosfamide-induced liver injury in adult male albino rat
}

\author{
Original \\ Article \\ Seham Abd El Hamid El Kalawy, Mohammad Hafez Ahmad Mostafa, Lamiaa \\ Ibrahim Abd El Fattah and Samaa Samir Ibrahim Kamar
}

Department of Medical Histology, Faculty of Medicine, Cairo University, Cairo, Egypt

\begin{abstract}
Background and Objectives: GIfosfamide (IFO) is a highly effective chemotherapeutic agent used for treating a variety of cancer but has reported to cause certain side effects such as hepatotoxicity. Being antioxidant and anti-inflammatory agents, omega-3 fatty acids ( $\omega 3$ FAs) were effective in the treatment of various medical conditions. This study aimed to elucidate the possible hepatoprotective role of $\omega 3$ FAs against IFO-induced liver injury.

Methods and Results: A total of 42 adult male albino rats were divided into 4 groups; the control group (Gp I) of 12 rats, and 3 groups each of 10 rats. Omega-3 group (Gp II) received $300 \mathrm{mg} / \mathrm{kg}$ /day of omega-3 plus orally. IFO group (Gp III) received intraperitoneal injection of IFO $50 \mathrm{mg} / \mathrm{kg}$, which was subdivided equally into IFO subgroup (Gp IIIa) and IFO recovery subgroup (Gp IIIb). IFO + Omega-3 group (Gp IV) received omega-3 plus as pretreatment and combined treatment with IFO. Liver sections were stained with Hematoxylin and Eosin (H\&E), Periodic acid Schiff (PAS) and immunohistochemical stain for caspase-3. The mean area percent (\%) of glycogen \& optical density of immunopositive cells were measured. IFO had degenerative effects on rat hepatocytes with marked depletion of glycogen, inflammatory cells infiltration, dilatation and congestion of blood sinusoids, prominent Kupffer cells (KC), besides, a significant increase in caspase-3 immunoexpression. However, these alterations were far less pronounced in IFO + Omega-3 group.

Conclusions: Administration of omega-3 FAs as a pretreatment and combined treatment with IFO ameliorated the liver injury induced by IFO indicating its hepatoprotective effects.
\end{abstract}

Key Words: Caspase 3, Ifosfamide, Liver, : Omega-3 FAs

Revised: 21 June 2017, Accepted: 10 October 2017

Corresponding Author: Samaa Samir Kamar, Department of Medical Histology, Faculty of Medicine, Cairo University, Cairo, Egypt Tel.: +2/01008909069, E-mail: Samaakamar@yahoo.com

ISSN: 2536-9172, Vol.1, No.2

\section{INTRODUCTION}

Being the predominant site for the biotransformation of drugs, the liver is a primary target of adverse drug reactions ${ }^{[1]}$. Liver injury is triggered by offending drugs, or more commonly, drug metabolites. The injury could be propagated by subsequent cell stress, accumulation of reactive oxygen species (ROS) and/or specific immune reactions, or directly by binding of metabolites to enzymes, lipids, nucleic acids or other cellular structures ${ }^{[2]}$.

Ifosfamide, a highly effective chemotherapeutic agent, is often used for treating a variety of pediatric and adult solid tumors ${ }^{[3]}$. However, it can have serious adverse effects such as hepatotoxicity ${ }^{[4]}$ and nephrotoxicity ${ }^{[5]}$.

Omega-3 fatty acids are long chain polyunsaturated fatty acids of plant and marine origin. They are of value in the treatment of various medical conditions as arthritis, asthma, inflammatory bowel disease and cardiovascular diseases ${ }^{[6]}$. They are capable of alleviating chemically induced acute hepatitis ${ }^{[7]}$, reversing hepatic steatosis and ameliorating pathophysiological features of non-alcoholic steatohepatitis such as hepatocellular damage and lobular inflammation ${ }^{[8]}$. Omega-3 FAs have been suggested to act as anti-oxidant and anti-apoptotic agents ${ }^{[9]}$.

In the current research, we aimed to elucidate the possible hepatoprotective role of omega-3 FAs against IFO-induced liver injury.

\section{PATIENTS AND METHODS}

Drugs:

Holoxan 1g (sterile Ifosfamide) (Baxter Oncology $\mathrm{GmbH}$, Germany): in the form of a vial containing a white crystalline powder that was dissolved in $25 \mathrm{ml}$ sterile distilled water. It was given at a dose of $50 \mathrm{mg} / \mathrm{kg}$ once daily by intraperitoneal injection ${ }^{[10]}$.

Omega-3 Plus (SEDICO, 6 October City, Egypt): used as gelatinous capsules, each contains $1000 \mathrm{mg}$ fish oil (eicosapentaenoic acid "EPA" \& docosahexaenoic acid 
"DHA" 30\%). Each capsule was evacuated by syringe carefully and was given orally at a dose of $300 \mathrm{mg} / \mathrm{kg}$ Omega- 3 plus once daily by an intra-gastric tube ${ }^{[11]}$.

\section{Animals:}

Forty two adult male albino rats weighing $150-200 \mathrm{gm}$ were divided into 4 groups. The animals were bred in the Animal House of Faculty of Medicine, Cairo University. Each group was housed in separate hygienic wire cage, in well ventilated room, fed ad libitum and allowed for free access to water. All procedures were held under the guidelines of animal care of Cairo University.

\section{Group I (Control group):}

Twelve rats were divided into:

- $\quad$ Subgroup Ia: 4 rats, each given $0.1 \mathrm{ml}$ saline daily orally using an intra-gastric tube for 20 days.

- $\quad$ Subgroup Ib: 4 rats, each given $0.25 \mathrm{ml}$ distilled water by intraperitoneal injection from day 16 to day 20 of the experiment.

- $\quad$ Subgroup Ic: 4 rats, each given combined oral saline using intra-gastric tube and intraperitoneal injection of distilled water as subgroups Ia and $\mathrm{Ib}$.

Three rats were sacrificed with each experimental group (II, IIIa, IIIb and IV)

\section{Group II (Omega-3 group):}

Ten rats, each received a single daily oral dose of Omega-3 plus $300 \mathrm{mg} / \mathrm{kg} / \mathrm{d}$ for 20 days.

\section{Group III (Ifosfamide group):}

Ten rats each received, starting on day 16 , a single daily intra-peritoneal injection of ifosfamide at a dose of $50 \mathrm{mg} /$ $\mathrm{kg}$ for 5 consecutive days.

They were divided into the following subgroups: the drug.

- $\quad$ IIIa: 5 rats were sacrificed 24 hours after stopping

- $\quad$ IIIb (recovery group): 5 rats were sacrificed 4 weeks after stopping the drug.

\section{Group IV ( Ifosfamide + Omega-3 group):}

Ten rats, each received a single daily oral dose of Omega-3 plus for 15 days, followed by both intraperitoneal injection of ifosfamide and a single oral dose of Omega-3 plus for 5 consecutive days, in the same doses as groups II \& III.

\section{A) Histological Study:}

All animals were sacrificed using isoflurane inhalation 24 hours after stopping the drug except group IIIb in which the rats were sacrificed 4 weeks after stopping IFO. Liver specimens were obtained by midline laparotomy and immediately fixed in 10\% formol saline for 24 hours. Paraffin blocks were prepared and $5 \mu \mathrm{m}$ thick sections were subjected to hematoxylin and eosin (H\&E) staining ${ }^{[12]}$.

\section{B) Histochemical Study:}

Periodic acid-Schiff(PAS ${ }^{[13]}$ reaction with hematoxylin counterstain for demonstration of glycogen content was performed.

\section{C) Immunohistochemical Study:}

Immunohistochemical staining ${ }^{[14]}$ using caspase-3 to detect apoptotic cells ${ }^{[15]}$. Ready to use rabbit polyclonal caspase 3 (CPP32) Ab-4 IgG (RB-1197-R7, Thermo Fisher Scientific; Fremont, USA) was used. Application of the primary (1ry) antibodies was followed by incubation in the humidity chamber for $60 \mathrm{~min}$ at room temperature. Tonsil was used as positive ( $+\mathrm{ve})$ control specimens. On the other hand, one of the liver sections was used as a negative (-ve) control by passing the step of applying the 1ry antibody.

\section{D) Morphometric Study:}

Using "Leica Qwin $500 \mathrm{C}$ " image analyzer (Cambridge, UK), assessment of mean area $\%$ of PAS positive (+ve) reaction and optical density of caspase-3 immunoexpression were performed in 10 non overlapping high power fields.

\section{E) Statistical Analysis:}

Quantitative data were summarized as means and standard deviations and compared using analysis of variance. The probability value $<0.05$ were considered statistically significant. Calculations were made on Statistical Package of Social Science software (SPSS), version 16 (Chicago, CA).

\section{RESULTS}

In the current study, no deaths were recorded.

\section{H\&E stained liver sections:}

Control subgroups (Gp I) revealed normal liver parenchyma with preserved architecture of hepatic lobules containing hepatocyte cords radiating from central veins with the portal tract at corners of ill-defined classic hepatic lobule. Hepatic cords were separated by blood sinusoids. Hepatocytes displayed eosinophilic cytoplasm and vesicular nuclei with prominent nucleoli 
(Fig. 1a). Omega-3 group (Gp II) revealed picture nearly similar to that of Gp I (Fig. 1b).

Liver sections of IFO subgroup (Gp IIIa) revealed marked vacuolated cytoplasm of swollen hepatocytes with small dark (pyknotic) nuclei, disturbed sinusoidal architecture in-between and dilated central vein (Fig. 1c). Inflammatory infiltration around the portal tact was noted with sporadic small foci of inflammatory cells. These changes were more evident in the periportal (zone 1) and midzonal (zone 2) areas (Fig. 1d).

In IFO recovery subgroup (Gp IIIb), most of hepatocytes displayed normal arrangement with acidophilic cytoplasm and vesicular nuclei. Some blood sinusoids were dilated and showed marked congestion with interstitial hemorrhage (Fig. 1e).

Ifosfamide and omega-3 group (Gp IV) showed apparently protected liver structure. The hepatic lobular architecture was preserved with apparently normal hepatocytes showing acidophilic cytoplasm and central vesicular nuclei. Mild dilatations of sinusoids were noted (Fig. 1f).

By higher magnification, Gp IIIa revealed hepatocytes exhibiting obvious vacuolated cytoplasm with dark pyknotic nuclei and some cells are devoid of nuclei (karyolysis) (Fig. 2a). Some pericentral areas (zone 3) still retain more or less normal hepatocytes and sinusoidal arrangement but with dilated lumen harboring prominent Kupffer cells (Fig. 2b). In Gp IIIb, some hepatocytes showed pyknotic nuclei or devoid of it, few necrotic areas were still evident. Multiple binucleated cells were seen (Figs. 2c\&d). Gp IV revealed numerous binucleated cells and Kupffer cells with few hepatocytes devoid of nuclei (Figs. 2e\&f)

\section{PAS stained liver sections:}

Strong PAS +ve reaction was seen in the cytoplasm of the hepatocytes throughout the different zones of the hepatic lobules in control rats (Fig. 3a). Gp II revealed a picture nearly similar to that of Gp I. While Gp IIIa showed marked depletion of PAS +ve glycogen granules in most of hepatic lobules sparing a small area around the central vein where liver cells were mildly affected (Fig. 3b). The recovery subgroup (Gp IIIb) showed mild to moderate PAS +ve reaction (Fig. 3c). Gp IV showed preservation of the glycogen contents in the cytoplasm of hepatocytes throughout the different zones of hepatic lobules (Fig. 3d).

Higher magnification of different zones of hepatic lobules in Gp III revealed that diminished PAS positivity in the hepatocytes was more evident away from the central vein (Fig. 4a). Besides, the depleted cells in midzonal areas and periportal areas showed fine PAS +ve granules in the cytoplasm mostly at the hepatic cell boundaries. The nuclei of hepatocytes showed abnormal PAS +ve reaction (Figs. 4b\&4c).

\section{Caspase-3:}

Liver sections of $\mathrm{Gp}$ I revealed almost negative immunoreaction for caspase-3 with few immunopositive cytoplasmic expressions in different zones (Figs.5a \& b). Gp II showed similar picture to Gp I. Gp IIIa revealed intense predominant cytoplasmic and few nuclear caspase-3 immunoreactivity of the hepatocytes. The vacuolated cells showed the strong brown immunoreactions in the cytoplasmic remnants and in few nuclei (Figs.5c \& d). While Gp IIIb showed mild caspase-3 +ve cytoplasmic immunoreactions (Fig.5e). In Gp IV, weak cytoplasmic caspase-3 immunoreactivity was noted (Fig. $5 \mathrm{f}$ ).

Table 1: The mean area $\%$ of PAS +ve reaction and the optical density of caspase-3 immuno-expression $( \pm \mathrm{SD})$ in the control and experimental groups

\begin{tabular}{|l|c|c|}
\hline Groups & $\begin{array}{c}\text { Mean Area \% of } \\
\text { PAS +ve reaction } \pm \text { SD }\end{array}$ & $\begin{array}{c}\text { Mean Optical Density of } \\
\text { Caspase-3 } \pm \text { SD }\end{array}$ \\
\hline Gp I & $75.08( \pm 3.8)$ & $0.42 \pm 0.02$ \\
\hline Gp II & $74.24( \pm 2.1)$ & $0.45 \pm 0.04$ \\
\hline GpIIIa & $38.05( \pm 4.5) *$ & $1.15 \pm 0.05^{*}$ \\
\hline GpIIIb & $53.77( \pm 4.2)$ & $0.90 \pm 0.07$ \\
\hline Gp IV & $60.02( \pm 3.1)$ & $0.48 \pm 0.03 \Theta$ \\
\hline
\end{tabular}

* Significant increase compared to other groups.

Ө Significant decrease compared to Gp IIIa and IIIb. 


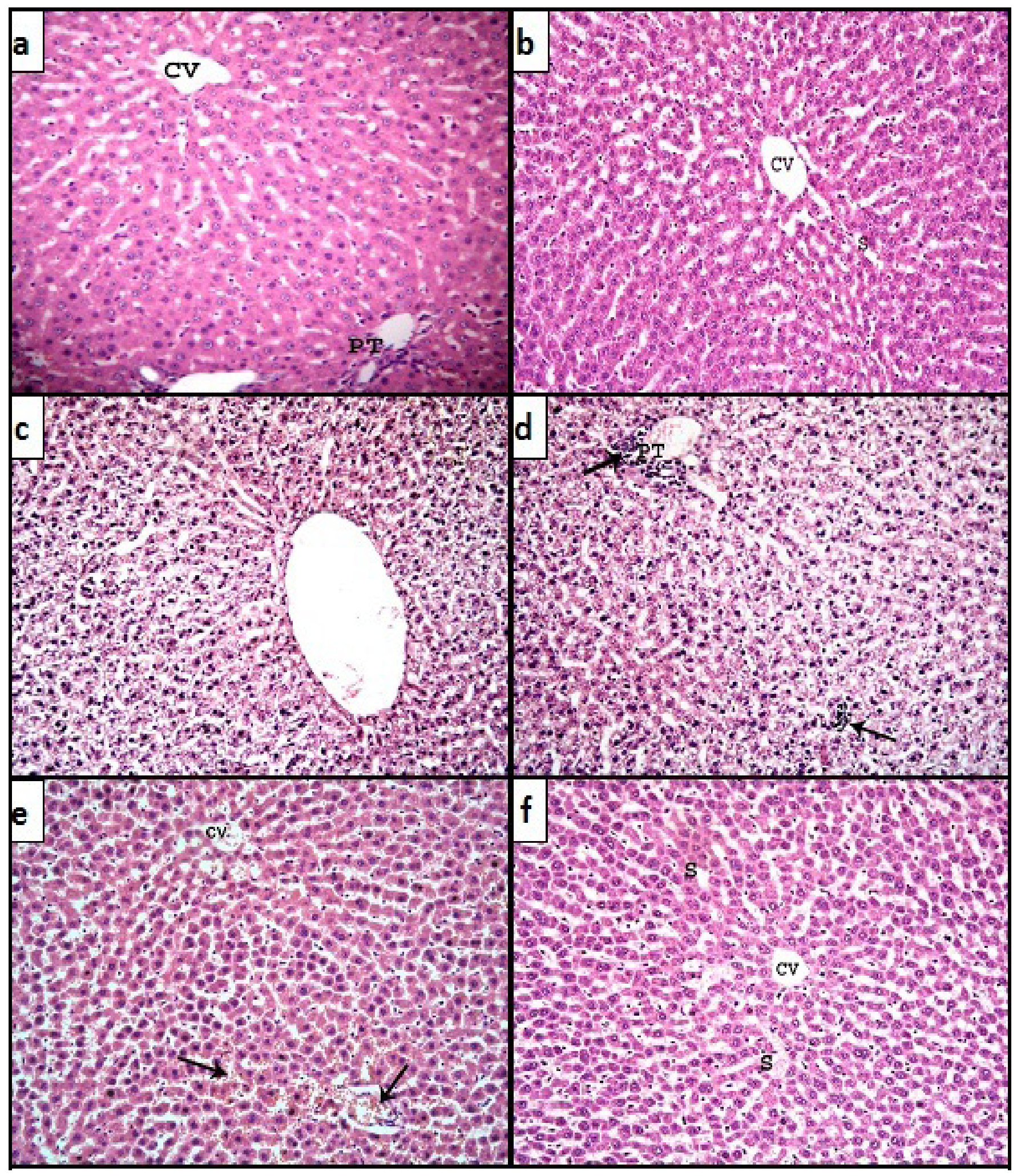

Fig. 1: Liver sections of control rats (a) showing normal architecture of hepatic lobule with hepatocytes arranged in plates radiating from the central vein $(\mathrm{CV})$ and the portal tract (PT) at corners of ill-defined classic hepatic lobule. Hepatic cords were separated by blood sinusoids. Hepatocytes showed eosinophilic cytoplasm and vesicular nuclei with prominent nucleoli. Gp II (b) showing hepatic lobule containing hepatocytes arranged in plates radiating from CV. The plates are separated by sinusoids (S). Gp IIIa (c) showing vacuolated hepatocytes with pyknotic nuclei radiating from dilated CV and (d) around PT (zone 1) and in midzonal area (zone 2). Inflammatory cell infiltration is present around the portal tract and in the midzonal area $(\rightarrow)$. Gp IIIb (e) showing preserved arrangement of hepatocytes around CV while parts of sinusoid are dilated and appear congested $(\rightarrow$ ). Gp IV (f) showing CV and radiating plates of hepatocytes that displayed acidophilic cytoplasm and vesicular nuclei. Mildly dilated blood sinusoids in between the hepatocytes can be detected (S). (H\&E x 200). 


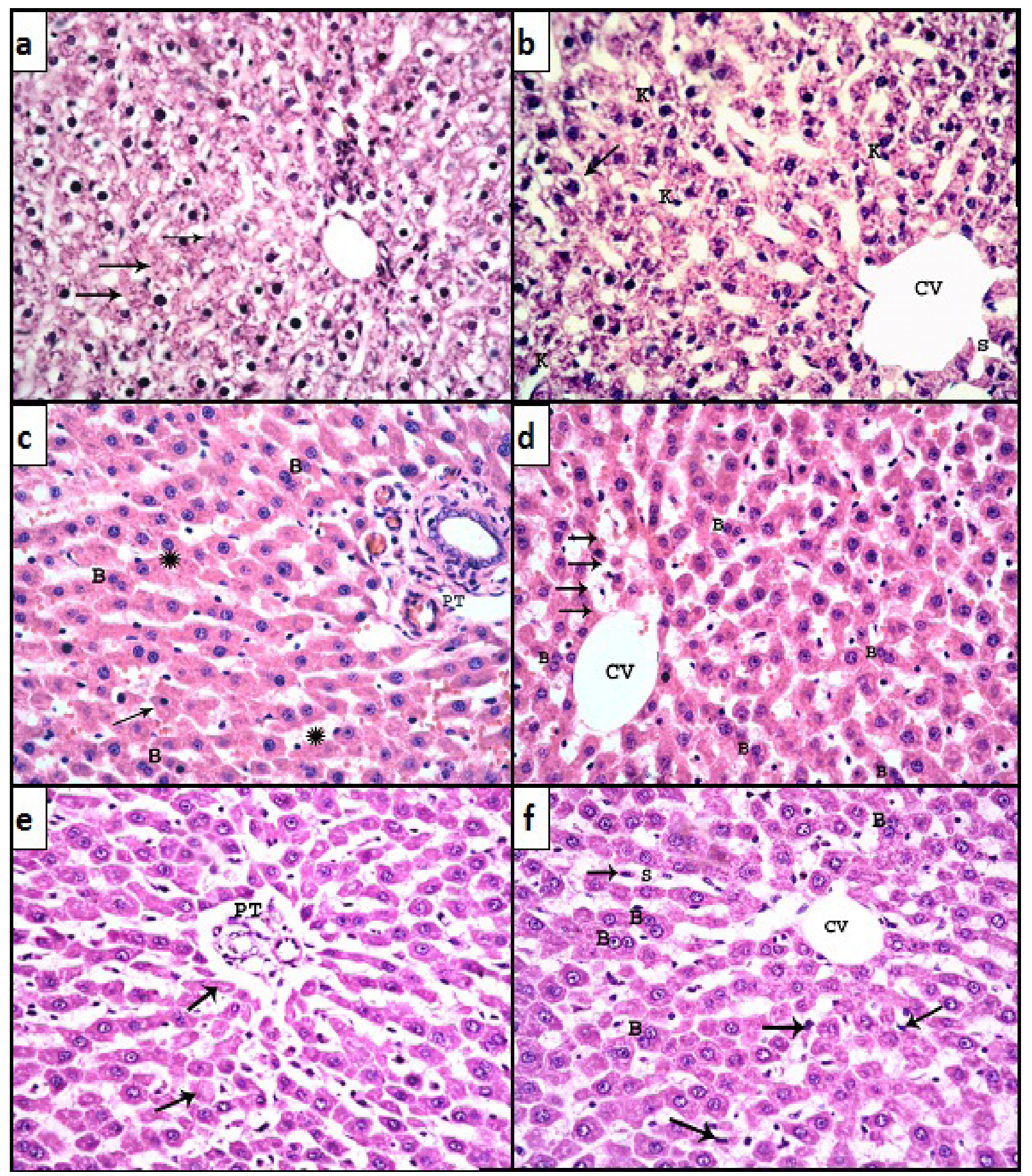

Fig. 2: Rat liver sections of GpIIIa (a) showing zone 1 revealed hepatocytes exhibiting obvious vacuolated cytoplasm with dark pyknotic nuclei and some cells are devoid of nuclei (karyolysis) $(\rightarrow$ ) and (b) showing the pericentral area (zone 3) still retain less vacuolated hepatocytes and normal sinusoidal arrangement. The hepatocytes away from CV show marked cytoplasmic vacuolation and having pyknotic nuclei $(\rightarrow)$. Dilated blood sinusoids (S) open into CV and harbor prominent Kupffer cells (K). GpIIIb (c) showing PT and hepatocytes with vesicular nuclei. Some hepatocytes show pyknotic eccentric nuclei $(\rightarrow)$ or devoid of it $(*)$. Numerous binucleated cells could be noted (B). Gp IIIb (d) showing dilated CV and necrotic areas $(\rightarrow$ ). Note the presence of numerous binucleated cells (B). Gp IV (e) showing PT with surrounding hepatocytes displaying vesicular nuclei. Few hepatocytes devoid of nuclei $(\rightarrow$ ). Gp IV (f) showing CV with radiating hepatocytes and blood sinusoids (S) in between, lined by prominent Kupffer cells $(\rightarrow)$. The hepatocytes displayed acidophilic cytoplasm and vesicular nuclei.Note the presence of numerous binucleated hepatocytes (B). (H\&E x 400). 


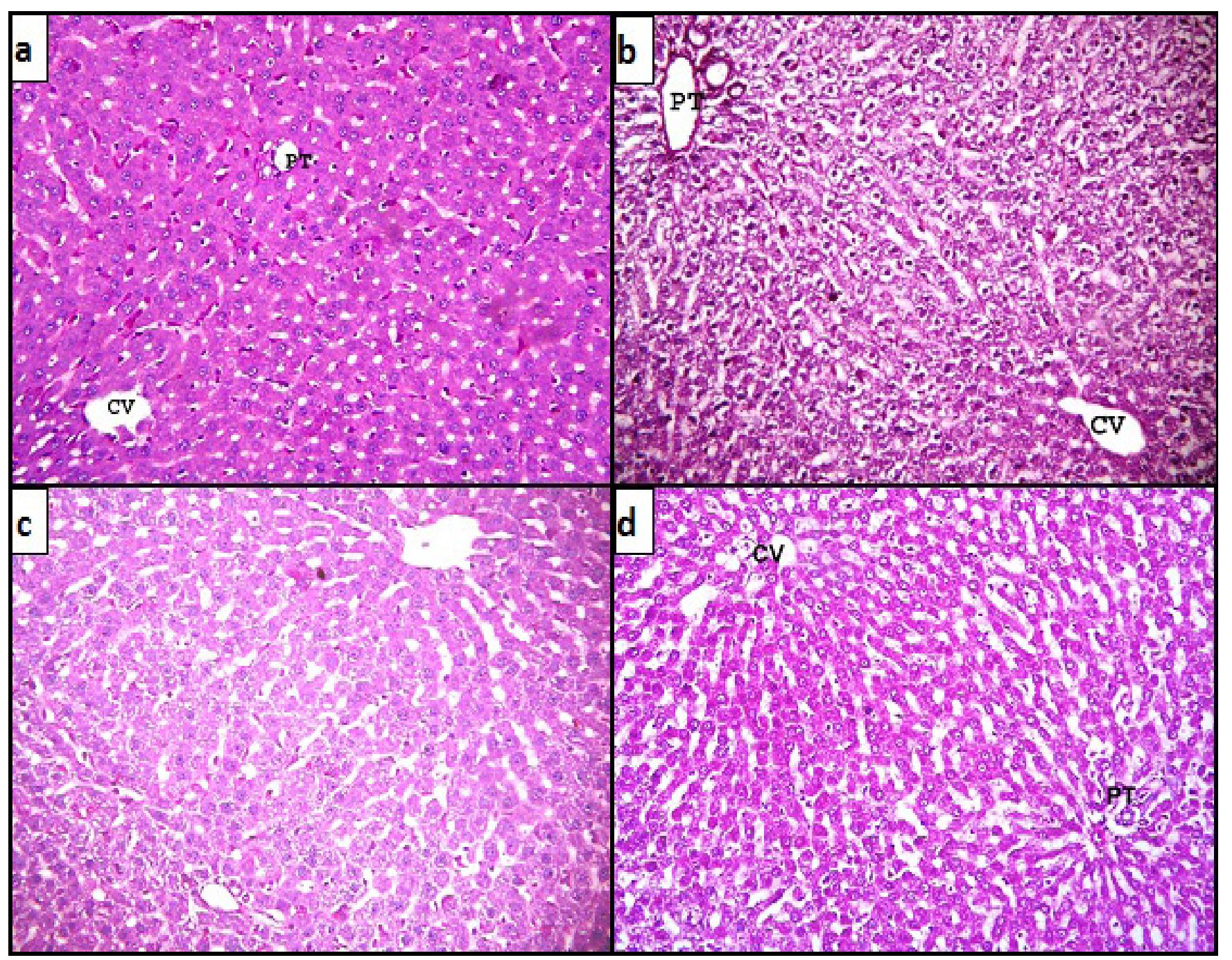

Fig. 3: Liver section of Gp I(a) showing uniform distribution of PAS +ve reaction in the cytoplasm of hepatocytes surrounding PT and CV. (b) Gp IIIa showing diminished PAS +ve reaction in hepatocytes surrounding PT. PAS positivity is slightly seen in hepatocytes radiating from CV. (c) Gp IIIb showing hepatocytes with mild to moderate PAS reaction. (d) Gp IV showing preservation of the uniform distribution of glycogen in hepatocytes radiating from CV and surrounding PT. (PAS x 200).

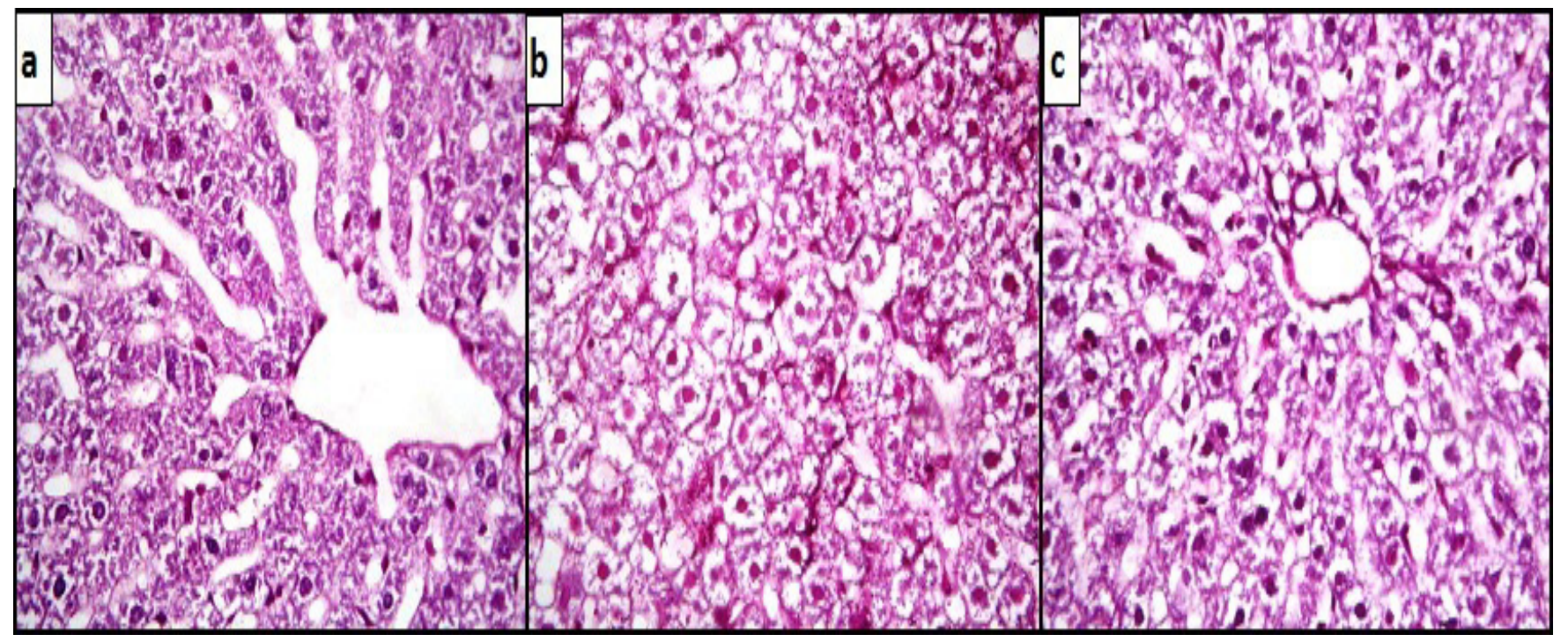

Fig. 4: Liver sections of GpIIIa showing: (a) diminished PAS positivity in the hepatocytes, more evident away from the central vein.(b) mild PAS reaction at the hepatic cell boundaries and abnormal PAS +ve reaction of nuclei.(c) diminished PAS positivity in the cytoplasmic remnants of the hepatocytes and abnormal PAS reaction of the nuclei with disturbed sinusoidal arrangement. (PAS x 400). 


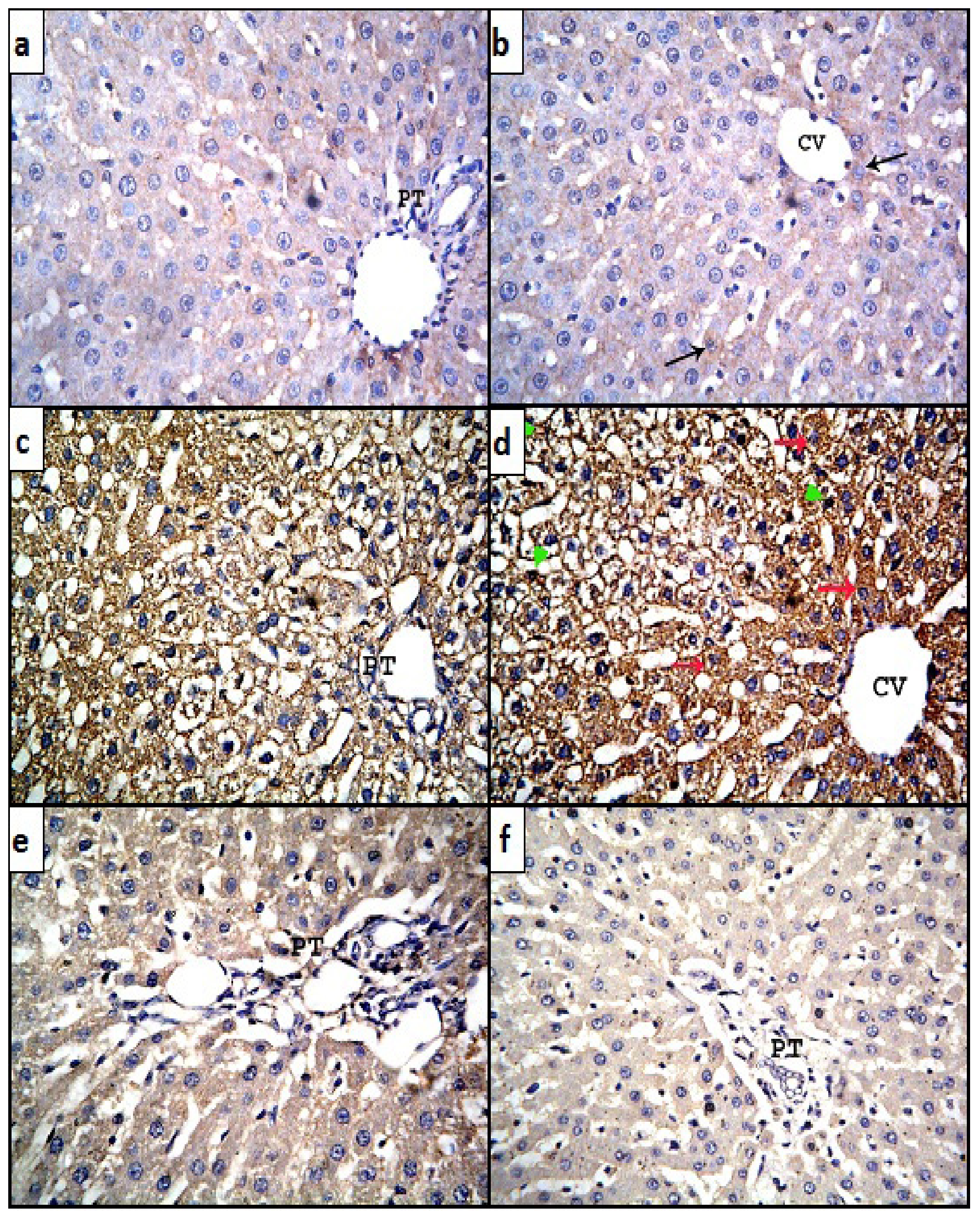

Fig. 5: Rat liver sections of Gp I showing (a) negative immune reaction for caspase-3 in the hepatocytes surrounding PT with(b) few immuno-stained cells are observed $(\rightarrow)$. Gp IIIa showing (c) intense immunoreactivity for caspase-3 in the cytoplasmic remnants of the vacuolated hepatocytes surrounding PT and (d) radiating from CV (red arrows) with few +ve nuclei (green arrowheads). (e) GpIIIb showing PT with hepatocytes exhibiting mild caspase-3 cytoplasmic immunoreactivity with vesicular nuclei. (f) Gp IV showing a weak cytoplasmic +ve caspase-3 immunoreactivity around PT. (Caspase-3 x 400). 


\section{DISCUSSION}

This study was an attempt to detect the histopathological changes produced in liver by IFO and elucidate the possible protective effects of omega-3 FAs as a pretreatment and a combined treatment on the changes which occurred in IFO-induced liver injury of adult male albino rats.

Histological examination of liver sections of omega-3 group (Gp II) revealed a picture similar to that of the control group; reflecting and confirming the lack of injury on the administration of omega-3 FAs solely.

Intra-peritoneal injection of IFO for 5 consecutive days produced markedly swollen vacuolated hepatocytes with pyknotic nuclei. Infiltration with inflammatory cells, dilatation of blood sinusoids, prominent Kupffer cells in addition to disturbed liver architecture. Alike, Takahashi et al. ${ }^{[16]}$ reported that IFO induces acute liver injury whereas $45 \%$ of the therapeutic dose of IFO is metabolized into chloroacetaldehyde (CAA) which causes increased formation of ROS and enhanced lipid peroxidation rate. In addition, it collapsed the mitochondrial membrane, induced the release of cytochrome $\mathrm{C}$ to the cytosol and significantly reduced the cellular ATP level that triggered cell death.

Moreover, the metabolism of CAA leads to increased ROS formation which target mitochondria and lysosomes and causes oxidative stress membrane damages in both organelles. Finally, mitochondrial/ lysosomal toxic cross talk potentiates oxidative stress that accelerates CAA hepatocyte cytotoxicity ${ }^{[17]}$. Also, the relationship between IFO and ROS can explain the cell injuries; as the resultant oxidative stress readily damages the biological molecules and ultimately induces cell death either by apoptosis or necrosis ${ }^{[18]}$.

The markedly swollen hepatocytes recorded in the current work might represent ballooning degeneration. Such swelling occurred as a result of dilatation of endoplasmic reticulum, which was the consequence of mitochondrial dysfunction and ATP depletion, leading to loss of ion homeostasis and plasma membrane integrity with massive influx of water and $\mathrm{Na}+$. The cytoplasm is partially rarefied, particularly along the cellular periphery, and the cytoplasmic cytoskeleton clumps around the nucleus ${ }^{[19]}$.

In present work, the distribution of degeneration mainly occupied the periportal (zone 1) and midzonal (zone 2) areas. This might be due to the fact that cells in zone 1 are the first to receive toxins from the sinusoidal blood and the first to show morphologic changes after exposure to drug toxins ${ }^{[20]}$
The inflammatory cell infiltration seen in the portal triads and the mid zonal area could be explained on the basis of that chemotherapy increased ROS generation and increased cell death which in turn initiate an inflammatory response ${ }^{[21]}$.

In the present study, the prominent $\mathrm{KC}$ could be correlated with the amount of injury to the hepatic tissue induced by IFO intoxication and represents a defensive mechanism of detoxification. Kupffer cell hyperplasia was established as a contributor to hepatic oxidative stress $^{[22]}$.

In the recovery subgroup (Gp IIIb), partial recovery was evident in the form of normal arrangement of hepatocytes with acidophilic cytoplasm and vesicular nuclei. Multiple binucleated cells were increased but the blood sinusoids were dilated and congested with interstitial hemorrhage. Few spots showed necrotic areas. Some hepatocytes showed pyknotic nucleus or karyolysis. In context, Bortolin et al. ${ }^{[23]}$ recorded binucleation as consequence of cell injury followed by regenerative growth after injury ${ }^{[23]}$.

Approximately protected liver structure was detected in Gp IV. The hepatic architecture was preserved showing hepatocytes with vesicular nuclei in a part of few cells devoid nuclei. Numerous binucleated cells and $\mathrm{KC}$ were detected. Although mild dilatation of blood sinusoids was noticed, no congestion was recorded. The detected hepatoprotective effect of omega- 3 could be explained by the fact that $\omega 3$ FAs from fish are enriched in EPA and DHA which have anti-inflammatory, antioxidant, antiapoptotic effects and regulate cell death. These findings were in accordance with Hiwa and Falah, ${ }^{[10]}$ who reported the protective role of fish oil against the side effects of IFO including hepatotoxicity, nephrotoxicity, changes in hematological parameters and the oxidative stress .

Naqshbandi et al. ${ }^{[2]}$ attributed the hepatoprotective effects of fish oil against ROS to its intrinsic biochemical and natural antioxidant properties. Wassall and Stillwell ${ }^{[25]}$ reported that when $\omega 3$ FAs are consumed, they are incorporated into cell membranes in all tissues of the body. This has profound effects on cell functions because the membrane lipids serve as a source of precursors for the synthesis of important signaling molecules involved in cell growth and development as well as modulation of inflammation. Omega-3 FAs appeared to affect the membrane organization and functions by altering membrane fatty acid composition, activating the endogenous antioxidant defense mechanism and accelerating the repair and/or regeneration of injured organelles, such as mitochondria and peroxisomes ${ }^{[26]}$. Also, DHA affects the cell membrane contents especially the phospholipids fluidity and modulate the receptor functions ${ }^{[27]}$. 
Concerning the apparent decrease of inflammatory cell infiltration in $\mathrm{Gp} \mathrm{IV}, \mathrm{Oh},{ }^{[28]}$ confirmed that DHA interact with several receptors, functioning as either an agonist or an antagonist in signaling responses. These receptors include plasma membrane bound Toll-like receptors (TLR) that play a significant role in innate and adaptive immune responses and the G-coupled protein receptor (GPR) specifically, GPR120 which is highly expressed on pro-inflammatory macrophages. The activation of GPR120 in macrophages by DHA is responsible for the inhibition of both TLR and TNFalpha mediated signaling pathways.

Resolvins and protectins are anti-inflammatory lipid mediators derived from $\omega 3$ FAs. They reduce tumor necrosis factor (TNF) expression and IFN- $\gamma$ production, promotion of $\mathrm{T}$-cell apoptosis reducing neutrophil activity and stimulating the uptake of apoptotic polymorphonuclear leukocyte ${ }^{[29]}$. In addition, they stimulate macrophage switching from the proinflammatory M1 phenotype to the anti-inflammatory M2 phenotype ${ }^{[30]}$.

Using PAS with hematoxylin counterstain, liver sections of Gp II revealed a picture nearly similar to that of Gp I whereas the hepatocytes possessed strong PAS +ve reaction in the cytoplasm, indicating the presence of glycogen.

In Gp IIIa, there was marked depletion of glycogen in hepatocytes away from the central vein. The mean area \% of PAS +ve reaction of Gp IIIa showed significant decrease compared to Gp I. Glycogen depletion noticed in the liver may be attributed to the oxidative stress and mitochondrial dysfunction caused by IFO. The oxidative stress leads to depression in the cytochrome oxidase and dehydrogenase enzymes activities $^{[31]}$ and this was reflected on the liver glycogen through enhancing glycogenolysis ${ }^{[32]}$.

Also in Gp IIIa, the depleted cells showed fine PAS + ve granules in the cytoplasm mostly at the hepatic cell boundaries, in addition to abnormal PAS +ve reaction of the nuclei. This could be explained by the toxic effect of IFO mustard and oxidative stress on the nucleic acid that breaks DNA strands and exposed the aldehyde groups on the DNA sugar ${ }^{[33]}$, resulted in abnormal reaction with the Schiff's reagent to produce red magenta color of the nucleus resembling the Feulgen reaction.

Group IIIb showed mild to moderate PAS +ve reaction that was significantly increased when compared with Gp IIIa, however it was less than that of Gp I. This could be explained by that during hepatic regeneration there was glycogen depletion ${ }^{[34]}$.

Gp IV showed preservation of the glycogen content in the cytoplasm of hepatocytes. The mean area $\%$ of PAS + ve reaction of Gp IV was significantly increased when compared to Gp IIIa, which may be due to the hepatoprotective effect of the omega-3 FAs shown in this study. Such effect can be attributed to the antioxidant effect of omega-3 FAs which accelerated the repair and regeneration of injured organelles ${ }^{[26]}$. However, more time may be needed to restore the normal value of glycogen content.

Anti-caspase-3 antibody was used for immunohistochemical detection of apoptotic hepatocytes. The caspases form a caspase-cascade system that plays central role in the induction, transduction and amplification of intracellular apoptotic signals for cell fate determination, regulation of immunity, and cellular proliferation and differentiation ${ }^{[35]}$. Noteworthy, activation of caspases usually ranges between extrinsic (death receptormediated) and intrinsic (mitochondria-mediated) pathways $^{[36]}$.

In Gp I, few cells showed +ve caspase-3 immunoreactivity with predominantly cytoplasmic localization. These immunohistochemical results were consistent with Veit-Simon ${ }^{[37]}$ who demonstrated that caspase- 3 immunoreactivity could be identified in normal liver tissue. Immunohistochemical examination of liver sections of Gp II showed a picture nearly similar to that of Gp I.

Group IIIa revealed intense predominant cytoplasmic and/or few nuclear immunoreactivity of the hepatocytes. The vacuolated cells showed strong brown immunoreactions in the cytoplasmic remnants and/or in the nuclei. The mean optical density of caspase-3 immuno-expression in Gp IIIa increased significantly compared to Gp I.

Caspase-3 activation is considered as the central and final apoptotic marker enzyme for both mitochondrial intrinsic and death-domain receptordependent extrinsic pathways. Chemotherapeutic agents induced apoptosis are always mediated by the mitochondrial pathway leading to activation of the initiator caspase-9, which in turn activates the effector caspases- 3 and caspase- $7^{[38]}$.

In Gp IIIb, the Immunohistochemical examination of liver sections revealed mild caspase- 3 +ve immunoreactivity in the cytoplasm of the hepatocytes. In correlation with $\mathrm{H} \& \mathrm{E}$ results, the observation of caspase- 3 immunoreactivity in the absence of cell death could be referred to the nonapoptotic functions of caspase-3. During the compensatory proliferation healing process of tissues, caspase- 3 provides the final shape and size to tissues to gain their original cell numbers and has a role in the modulation of cell growth $^{[35]}$.

Weak caspase-3 immunoreactivity was noted in rat liver sections of Gp IV in different zones of the hepatic 
lobule. Gp IV mean optical density of caspase-3 immunoexpression showed significant decrease when compared with Gp IIIa and IIIb.

The antiapoptotic effect of EPA was evident from its ability to counteract the main biochemical markers of apoptosis such as the activation of caspases and the DNA fragmentation ${ }^{[39]}$.

\section{CONCLUSION}

Ifosfamide demonstrated serious hepatotoxic effects on the liver of adult male albino rats. The pretreatment and also the combined treatment of $\omega 3$ FAs with IFO ameliorated the liver injury of male albino rats indicating its hepatoprotective effects. Subsequently, $\omega 3$ FAs may be an effective supplementation to maximize the clinical use of IFO in the treatment of various malignancies with reduced hepatotoxicity.

\section{CONFLICT OF INTEREST}

The authors have no conflicting financial interest.

\section{REFERENCES}

1. Linling C, Qiang Y, Hao Y, Michael PH and Cynthia $\mathrm{J}$. Involvement of natural killer T cells in halothaneinduced liver injury in mice. Biochem Pharmacol (2010); 80(2): 255-261.

2. Russmann S, Kullak-Ublick GA and Grattagliano I. Current concepts of mechanisms in drug-induced hepatotoxicity. Curr Med Chem (2009); 16: 30413053.

3. Lam CG, Furman WL, Wang C, Spunt SL, Wu J, Ivy P, Santana VM and McGregor LM. Phase I clinical trial of ifosfamide, oxaliplatin and etoposide (IOE) in pediatric patients with refractory solid tumors. J Pediatr Hematol Oncol (2015); 37(1): e13-18.

4. Chhibar P, Zhu Z, Cheedella NK, Chaudhry R and Wang JC. Hepatitis B reactivation after ifosfamide therapy for retroperitoneal sarcoma. Am J Case Rep (2016); 1(17): 371-374.

5. Mhaidat NM, Ali RM, Shotar AM and Alkaraki AK. Antioxidant activity of simvastatin prevents ifosfamide-induced nephrotoxicity. Pak J Pharm Sci (2016); 29(2): 433-437.

6. Jain AP, Aggarwal KK and Zhang PY. Omega-3 fatty acids and cardiovascular disease. Eur Rev Med Pharmacol Sci (2015); 19(3): 441-445.

7. Schmöcker C, Weylandt KH, Kahlke L, Wang J, Lobeck H, Tiegs G, Berg T and Kang JX. Omega-3 fatty acids alleviate chemically induced acute hepatitis by suppression of cytokines. Hepatology (2007); 45(4):864-869.

8. Hendrik AM, Michal H, Jaap JK, Syert LN, Jochem RW, Aart JN , Fiebo JW, Jaap S and Thomas M. Reversal of hepatic steatosis by omega- 3 fatty acids measured non-invasively by $1 \mathrm{H}$-magnetic resonance spectroscopy in a rat model. $\mathrm{J}$ of Gastroentero and Hepato ( 2011 ); 26(2): 356-363.

9. Fahmy HA, Abd El Azim AS and Gharib OA. Protective Effects of omega-3 fatty acids and/ or nanoselenium on cisplatin and ionizing radiation induced liver toxicity in rats. IJPER (2016); 50(4):649-656.

10. Hiwa RA and Falah MA. Protective role of omega-3 fish oil against the toxicity of ifosfamide in male rats. Jordan J of Biolog Scie (2012); 5(1): 37-46.

11. Meganathan M, Gopal KM, Sasikala P, Mohan J, Gowdhaman N, Balamurugan K, Nirmala P, Santhakumari $\mathrm{S}$ and Samuel V. Evaluation of hepatoprotective effect of omega 3-fatty acid against paracetamol induced liver injury in albino rats. Global J of Pharmacol (2011); 5 (1): 50-53.

12. Kiernan JK. Histological and Histochemical methods In: Theory and practice. London, New York and New Delhi: Arnold Publisher; (2001). 111-162.

13. Bancroft JD and Gamble M. Theory and practice of histological techniques. Edinburgh: Churchill Livingstone; (2002).172-175.

14. Bancroft J D and Cook H C. Immunocytochemistry. In: Manual of Histological Techniques and their Diagnostic Applications. Edinburgh, London, Madrid, Melbourne, New York and Tokyo: Churchill Livingstone; (1994). 263-325.

15. Sun W, Su Q, Cao X, Shang B, Chen A, Yin H and Liu B. High Expression of Polo-Like Kinase 1 Is Associated with Early Development of Hepatocellular Carcinoma. Int J Genomics (2014); 312130-312138.

16. Takahashi K, Sakurai K, Tanaka H and Fujimoto Y. Necrotic pathway in human osteosarcoma Saos-2 cell death induced by chloroacetaldehyde. Anti-Canc Drug (2007); 18(5):543-553.

17. Jalal P, Hosseinia MJ, Eskandari MR and Rahmani F. Involvement of four different intracellular sites in chloroacetaldehyde-induced oxidative stress cytotoxicity. Iranian J of Pharmac Research (2012); 11 (1): 265-276.

18. Zhang T, Zhang Y, Cui M, Jin L, Wang Y, Lv F, Liu Y, Zheng W, Shang H, Zhang J, Zhang M, Wu H, Guo 
J, Zhang X, Hu X, Cao CM and Xiao RP. CaMKII is a RIP3 substrate mediating ischemia- and oxidative stress-induced myocardial necroptosis. Nat Med (2016); 22(2): 175-182.

19. Suriawinata AA and Thung SN. Hepatocyte degeneration, Death and Regeneration. In: Liver Pathology, an Atlas and Concise Guide. New York: Demos Medical Publishing, LLC (2011). 19-20.

20. Ross $\mathrm{MH}$ and Pawlina W. Liver, Gall Bladder and Pancrease. In: Histology a Text and Atlas. Philadelphia and London: Lippincott Williams \& Wilkins, a Wolters Kluwer (2011). 628-642.

21. Fahmi AN, Shehatou GS, Shebl AM and Salem HA. Febuxostat protects rats against lipopolysaccharideinduced lung inflammation in a dose-dependent manner. Naunyn Schmiedebergs Arch Pharmacol (2016); 389(3): 269-278.

22. Hassan KA, Ahmed MA, Hassanein KMA and Waly $\mathrm{H}$. Ameliorating effect of vitamin $\mathrm{C}$ and selenium against nicotine induced oxidative stress and changes of p53 expression in pregnant albino rats. Journal of Advanced Veterinary and Animal Research (2016); 3(4): 321-331.

23. Bortolin JA, Quintana HT, Tomé Tde C, Ribeiro FA, Ribeiro DA and de Oliveira F. Burn injury induces histopathological changes and cell proliferation in liver of rats. World J Hepatol (2016); 8(6):322-330.

24. Naqshbandi A, Khan W, Rizwan S and Khan F. Studies on the protective effect of fish oil against cisplatin induced hepatotoxicity. Biology and Medicine (2012); 3 (2): 86-97.

25. Wassall ST and Stillwell W. Polyunsaturated fatty acid-cholesterol interactions: Domain formation in membranes. Biochimica et Biophysica Acta (2009); 1788: 24-32.

26. Khan MW, Priyamvada S, Khan SA, Khan S, Naqshbandi A and Yusufi ANK. Protective effect of $\omega-3$ polyunsaturated fatty acids (PUFAs) on sodium nitroprusside-induced nephrotoxicity and oxidative damage in rat kidney. Human and Experimental Toxicology (2012); 1-15.

27. Guixà-González R, Javanainen $\mathrm{M}$, Gómez-Soler $\mathrm{M}$, Cordobilla B, Carles Domingo J, Sanz F, Pastor M, Ciruela F, Martinez-Seara H and Selent J. Membrane omega-3 fatty acids modulate the oligomerisation kinetics of adenosine $\mathrm{A} 2 \mathrm{~A}$ and dopamine $\mathrm{D} 2$ receptors. Sci. Rep (2016); 6:19839.

28. Oh DY, Talukdar $\mathrm{S}$, Bae EJ, Imamura $\mathrm{T}$, Morinaga H, Fan WQ, Li P, Lu WJ, Watkins SM and Olefsky
JM. GPR120 is an Omega-3 Fatty Acid Receptor Mediating Potent Anti-Inflammatory and Insulin Sensitizing Effects. Cell (2010); 142(5): 687-698.

29. Serhan $\mathrm{CN}$, Chiang $\mathrm{N}$ and Van Dyke TE. Resolving inflammation: dual anti-inflammatory and proresolution lipid mediators. Nat Rev Immunol (2008); 8(5): 349-361.

30. Titos E, Rius B, González-Périz A, López-Vicario C, Morán-Salvador E, Martínez-Clemente M, Arroyo $\mathrm{V}$ and Clària J. Resolvin D1 and its precursor docosahexaenoic acid promote resolution of adipose tissue inflammation by eliciting macrophage polarization toward an M2-like phenotype. J Immunol (2011); 187: 5408-5418.

31. Alfaradhi MZ, Fernandez-Twinn DS, MartinGronert MS, Musial B, Fowden A and Ozanne SE. Oxidative stress and altered lipid homeostasis in the programming of offspring fatty liver by maternal obesity. Am J Physiol Regul Integr Comp Physiol (2014); 307(1): R26-34.

32. Galal MG, Ibrahim SN, El-Sady EA and Hamdi KN. Histological and Histochemical Studies on the Toxic effect of hypervitaminosis A on the Liver and Spleen of the Albino Rat. Sci Med J Cai Med Synd (1991); 3(2): 171-186.

33. Minev BR. Alkylating Agents. In: Cancer Management in Man: Chemotherapy, Biological Therapy, Hyperthermia and supporting measures. London and New York: Springer Science and Business Media B.V., (2011). 61-86.

34. Haschek WM and Rousseaux CG. Hepatobiliary system. In: Handbook of toxicologic pathology. San Diag, New York, Boston, London, Sydney, Tokyo and Toronto: Academic Press Inc., Harcourt Brace Jovanovich Publisher; (1991). 280.

35. Chowdhury I, Tharakan B and Bhat GK. Caspases-An update. Comp Biochem Physiol B Biochem Mol Biol. (2008); 151: 10-27.

36. Ma Y, Zhu B, Yong L, Song C, Liu X, Yu H, Wang P, Liu Z and Liu X. Regulation of Intrinsic and Extrinsic Apoptotic Pathways in Osteosarcoma Cells Following Oleandrin Treatment. Int J Mol Sci (2016); 17(11): 1950-1962.

37. Veit-Simon E, Albrecht B, Wilfried B, Rolf $\mathrm{SH}$ and Michael S. Immunohistochemical detection of activated caspases in apoptotic hepatocytes in rat liver. Toxicologic Pathology (2004); 32: 9-15.

38. Shang J, Yang F, Wang Y, Wang Y, Xue G, Mei Q, Wang $F$ and Sun S. MicroRNA-23a antisense 
enhances 5-fluorouracil chemosensitivity through APAF-1/caspase-9 apoptotic pathway in colorectal cancer cells. J Cell Biochem (2014); 115(4):772-784.

39. Silvia C, Benedetta T, Flavio F, Claudia P, Annalisa F, Claudio S, Claudio MC and Carla P. Antiapoptotic and Antiautophagic Effects of Eicosapentaenoic Acid in Cardiac Myoblasts Exposed to Palmitic Acid. Nutrients (2012); 4: 78-90. 